\title{
Multi-Stage Adaptive Pool Testing Model with Test Errors; Improved Efficiency
}

\author{
Okoth Annette W. ${ }^{1}$, Nyongesa kennedy L. ${ }^{2}$, Kwach Bonface O. ${ }^{3}$ \\ ${ }_{1 ; 2}$ Department of Mathematics, Masinde Muliro University of Science and Technology \\ ${ }^{3}$ Department of Mathematics, Kibabii University
}

\begin{abstract}
Testing units one by one for presence or absence of a trait is expensive and time consuming. This study presents a multi-stage adaptive pool testing estimator ${ }^{\wedge} p n$ of prevalence of a trait in the presence of test errors, since errors in experiments are inevitable. Pool testing is more efficient, less expensive and less time consuming. An increase in the number of stages improves the efficiency of the estimator, hence construction of a multi-stage model. The study made use of the Maximum Likelihood Estimate (MLE) method and Martingale method to obtain the adaptive estimator and Cramer-Rao lower bound method to determine the variance of the constructed estimator. Mat lab and $R$, statistical softwares were used for Monte-carlo simulation and verification of the model, then analysis and discussion of properties of the constructed estimator in comparison with the non-adaptive estimator in the literature of pool testing done alongside provision of the con dence interval of the estimator. Results have shown that as the number of stages increases, the efficiency of the multistage adaptive estimator in the presence of test errors also increases in comparison with the non-adaptive estimator in the presence of test errors. This makes the multi-stage adaptive estimator better than the corresponding non-adaptive estimator in the literature of pool testing.
\end{abstract}

Keywords: Pool testing, Adaptive estimator, Confidence interval

\section{Introduction}

Prevalence of defective units in a large population from accurate diagnostic tests is a fundamental risk assessment and management factor. Estimation of defective units oneby-one is inefficient and uneconomical, considering that in a given population only a few individuals may be defective. It is against this background that pool testing comes in handy because it is more effective, less time consuming and less expensive [4]. Pool testing occurs when units from a population are pooled and tested as a group for the presence or absence of a particular trait. It also reduces the Mean Squared Error (MSE) of the estimates, hence it is more efficient, as was established by Sobel and Ellashoff, [11]. There are two forms of pool testing namely

(i) Non-adaptive pool testing scheme

(ii) Adaptive pool testing scheme

\subsection{Non-adaptive testing scheme}

In this testing scheme, a large population is divided in to $n$ groups which are then subjected to testing [4]. When tested, a group can either test positive or negative and the outcome of the test aids in constructing the non-adaptive model.

\subsection{Adaptive testing scheme}

In this scheme a population is divided in to $n$ groups, which are partitioned depending on the number of stages to be considered. Predetermined parameters are used to partition the groups and the number of partitioning parameters depends on the number of stages [8]. Partitioned groups are then tested at various stages for the presence or absence of a trait and the results used to construct the adaptive model. 


\subsection{Introduction of the model}

In this study we obtain a multi-stage adaptive estimator $\hat{p}_{n}$ of prevalence of a trait in the presence of test errors,using the maximum likelihood estimate (MLE) method and investigate its properties. The adaptive testing scheme involves testing groups in stages and updating group sizes from one stage to the next, with the group size at a stage depending on the outcome of the test(s) at the preceding stage(s). That is testing $n_{1}$ groups each of size $k_{1}$ at stage one; $n_{2}$ groups each of size $k_{2}$ at stage two; $n_{3}$ groups each of size $k_{3}$ at stage three and so on; where $k_{3}$ depends on both $k_{1}$ and $k_{2}$ while $k_{2}$ depends on $k_{1}$. For a general adaptive scheme, at stage $i n_{i}$ groups each of size $k_{i}$, where $k_{i}$ depends on $k_{i-1}, k_{i-2}, k_{i-3}, \ldots . . k_{1}$ are constructed. The constructed groups are then subjected to testing, where a group yields either a positive or a negative result. The number of groups, $n_{i}$ is determined before the experiment is carried out while $k_{i}^{\prime} s$ are sequentially determined as the experiment progresses.

\section{Literature Review}

Pool testing has been recognized as a sampling scheme that can provide substantial benefits [9]. Early application of pool testing include tests for prevalence of plant virus transmissions by insects [12] and [11] and this was one of the pioneering applications of this concept. In [4] statistical and mathematical concepts of pool testing are introduced and used to estimate the proportion of individuals infected with some disease among the US conscripts. He also derived optimum group sizes assuming that the population was large enough for the application of the binomial model and consequently realized significant savings by reducing the number of tests required. In [11] estimation in the pool testing procedure is discussed. In the subsequent years this concept has had relevant applications in various clinical studies including psychopathology, public health and plant quarantine [1] and [3]. Alternatively, positively pooled samples can be partitioned into relatively smaller subsets there by reducing on cost and effort, which provides obvious motive for pooling samples [8]. In [7] an estimation model based on pool testing with retesting pools that test negative is developed. Pool testing need not only be applied to population where retesting is needed [10], like in identification of disease infected individuals in a human population, but also on other populations with no intentions of retesting the individuals contributing to positive pooled samples. For instance if a bunch of food items is being tested for contamination, there may be no interest in identifying the particular items which are affected. The aim may instead be on estimating the proportion of defective items in a population or deciding that the number of positive pooled samples justifies removing a food product from the market. In another related study, bacteriological testing of egg laying hens of salmonella in Great Britain was carried out using organ cultures pooled five at a time. Individual samples contributing to positive pooled samples are not tested again. A population comprised of birds in a hen house. If the infection was confirmed they were destroyed and compensation paid for the number of birds estimated to be uninfected [10]. In this procedure maximum likelihood estimation is applied to estimate the proportion and Cramer-Rao lower bound method is used to determine the variance of the estimator. In this paper, we present a multi-stage adaptive pool testing model with imperfect tests, that is applicable to real life situations where there is a possibility of errors due to test kits. 


\section{Model Description Formulation and Analysis}

We describe a multi-stage adaptive scheme with imperfect tests as it is the backbone of this study and thereafter perform comparison analysis with other existing estimators, both in the presence and absence of test errors. For a multi-stage adaptive scheme, we set $n_{1}=\lambda_{1} n, n_{2}=\lambda_{2} n, n_{3}=\lambda_{3} n, \ldots ., n_{n}=\left(1-\lambda_{1} n-\lambda_{2} n-\ldots .-\lambda_{n-1} n\right)$; where $\lambda_{1}$, $\lambda_{2}, \ldots . ., \lambda_{n-1}$ are parameters used to partition the pools; $k_{2}$ depends on the outcome at stage $1, k_{3}$ depends on the outcomes at stages 1 and 2 and $k_{n}$ depends on the outcomes at stages $1,2,3, \ldots \ldots ., n-1$. Each constructed group at each stage is then subjected to testing, yielding either a positive or negative result. This is shown in Figure 3.2 below:

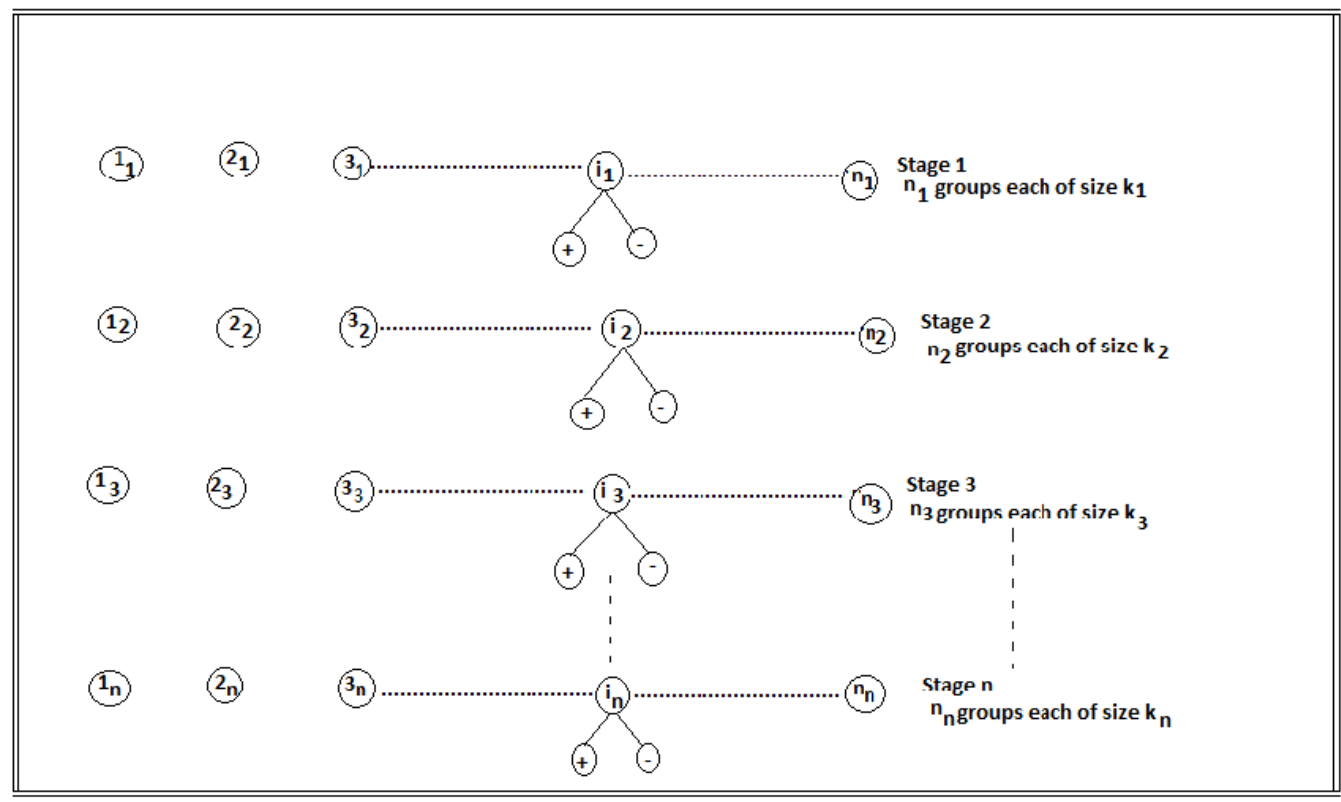

Figure 3.2: Multi-stage adaptive pool testing.

To achieve the construction of the multi-stage adaptive model in the presence of test errors, we consider two stage, three stage and four stage adaptive models in the presence of test errors and there after generalize to obtain the multi-stage model.

\subsection{Two stage adaptive model}

In this scheme, the population is divided into two sets of groups $n_{1}$ and $n_{2}$ which are tested in two stages, with $n_{1}$ groups tested at stage one and $n_{2}$ groups tested at stage two. We set $n_{1}=\lambda n$ and $n_{2}=(1-\lambda n)$, where $n$ is the number of groups constructed initially. $k_{1}$ which is the group size at stage one is determined by

$$
k_{1}=\left.\operatorname{argmin}_{l}[\operatorname{Var}(\hat{p})]\right|_{p=p_{0}},
$$

Suppose $X_{1}$ groups test positive on the test at stage-one, then

$$
X_{1} \sim \operatorname{Binomial}\left(\lambda n,\left.\pi(p)\right|_{k=k_{1}}\right) .
$$

where $\lambda$ is the parameter used to partition the pools while $\pi(p)$ istheprobabilitythatagroupisdefectived $\eta\left[1-(1-p)^{k}\right]+(1-\phi)(1-p)^{k}(3)$ Using this model we obtain the prevalence estimator at stage one as

$$
\hat{p}_{1}=1-\left[\frac{\eta-\frac{X_{1}}{\lambda n}}{\eta+\phi-1}\right]^{\frac{1}{k_{1}}},
$$


The variance of Equation (4) is similar to the variance of the non-adaptive estimator in the absence of test errors, $\hat{p}$ except for $K_{1}$ in place of $p$. This variance is given by

$$
\begin{aligned}
\operatorname{Var}(\hat{p}) & =\frac{\pi(p)(1-\pi(p))}{n k^{2}(1-p)^{2 k-2}(\eta+\phi-1)^{2}} \\
& =\frac{(1-p)^{2-2 k} \pi(p)(1-\pi(p))}{n k^{2}(\eta+\phi-1)^{2}} \\
& =\frac{(1-p)^{2} \pi(p)(1-\pi(p))(1-p)^{-2 k}}{n k^{2}(\eta+\phi-1)^{2}}
\end{aligned}
$$

For the estimator at stage two, $\hat{p}_{2}$, we have $\lambda n$ groups each of size $k_{1}$ tested at stage one and $1-\lambda n$ groups each of size $k_{2}$ tested at stage two. $k_{2}$ is determined by

$$
k_{2}=\left.\operatorname{argmin}_{l}\left[\operatorname{Var}\left(\hat{p}_{1}\right)\right]\right|_{p_{1}=p},
$$

Suppose that out of the $(1-\lambda) n$ groups each of size $k_{2}$ tested at stage two, $X_{2}$ groups test positive on the test, then for fixed $X_{1}$ we have

$$
X_{2} \mid X_{1} \sim \operatorname{Binomial}\left((1-\lambda) n, \pi_{2 \mid 1}(p)\right)
$$

Using this model, the estimator at stage two can be obtained as the solution to

$$
\begin{aligned}
& \frac{k_{1} X_{1} q^{k_{1}}[(1-\phi)-\eta]}{\eta-(\eta+(1-\phi)) q^{k_{1}}}+\frac{k_{2}\left(X_{1}\right) X_{2} q^{k_{2}\left(X_{1}\right)}[(1-\phi)-\eta]}{\eta-(\eta+(1-\phi)) q^{k_{2}\left(X_{1}\right)}} \\
= & \frac{k_{1} q^{k_{1}}\left(\lambda n-X_{1}\right)(\eta+(1-\phi))}{1-\left[\eta-(\eta+(1-\phi)) q^{k_{1}}\right]}+\frac{k_{2}\left(X_{1}\right) q^{k_{2}\left(X_{1}\right)}\left[(1-\lambda) n-X_{2}\right][\eta+(1-\phi)]}{1-\left[\eta-(\eta+(1-\phi)) q^{k_{2}\left(X_{1}\right)}\right]} .
\end{aligned}
$$

and using cramer-Rao lower bound, its variance is obtained as

$$
\operatorname{Var}\left(\hat{p}_{2}\right)=\frac{\pi_{1}(p) \pi_{2}(p)\left(1-\pi_{1}(p)\right)\left(1-\pi_{2}(p)\right)}{A},
$$

where $A$ is defined in the appendices.

\subsection{Three stage adaptive model}

Next we consider the estimator at stage three, $\hat{p}_{3}$, where we have $\lambda_{1} n$ groups each of size $k_{1}$ tested at stage one, $\lambda_{2} n$ groups each of size $k_{2}$ tested at stage two and $1-\lambda_{1} n-\lambda_{2} n$ groups each of size $k_{3}$ tested at stage three. $k_{3}$ is determined by

$$
k_{3}=\left.\operatorname{argmin}_{l}\left[\operatorname{Var}\left(\hat{p}_{2}\right)\right]\right|_{p_{2}=p_{1}},
$$

If out of the $\left(1-\lambda_{1}-\lambda_{2}\right) n$ groups each of size $k_{3}$ tested at stage three, $X_{3}$ groups test positive on the test, then for fixed $X_{1}$ and $X_{2}$ we have

$$
X_{3} \mid X_{1}, X_{2} \sim \operatorname{Binomial}\left(\left(1-\lambda_{1}-\lambda_{2}\right) n, \pi_{3 \mid 1,2}(p)\right)
$$

We use this model to obtain the estimator at stage three as the solution to

$$
\begin{aligned}
& \frac{k_{1} X_{1} q^{k_{1}}[(1-\phi)-\eta]}{\eta-(\eta+(1-\phi)) q^{k_{1}}}+\frac{k_{2}\left(X_{1}\right) X_{2} q^{k_{2}\left(X_{1}\right)}[(1-\phi)-\eta]}{\eta-(\eta+(1-\phi)) q^{k_{2}\left(X_{1}\right)}}+\frac{k_{3}\left(X_{1}, X_{2}\right) X_{3} q^{k_{3}\left(X_{1}, X_{2}\right)}[(1-\phi)-\eta]}{\eta-(\eta+(1-\phi)) q^{k_{3}\left(X_{1}, X_{2}\right)}} \\
= & \frac{k_{1} q^{k_{1}}\left(\lambda_{1} n-X_{1}\right)(\eta+(1-\phi))}{1-\left[\eta-(\eta+(1-\phi)) q^{k_{1}}\right]}+\frac{k_{2}\left(X_{1}\right) q^{k_{2}\left(X_{1}\right)}\left[\lambda_{2} n-X_{2}\right][\eta+(1-\phi)]}{1-\left[\eta-(\eta+(1-\phi)) q^{k_{2}\left(X_{1}\right)}\right]} \\
& +\frac{k_{3}\left(X_{1}, X_{2}\right) q^{k_{3}\left(X_{1}, X_{2}\right)}\left[\left(1-\lambda_{1}-\lambda_{2}\right) n-X_{3}\right][\eta+(1-\phi)]}{1-\left[\eta-(\eta+(1-\phi)) q^{k_{3}\left(X_{1}, X_{2}\right)}\right]}=0,
\end{aligned}
$$


and its variance as

$$
\operatorname{Var}\left(\hat{p}_{3}\right)=\frac{\pi_{1}(p) \pi_{2}(p) \pi_{3}(p)\left(1-\pi_{1}(p)\right)(p)\left(1-\pi_{2}(p)\right)(p)\left(1-\pi_{3}(p)\right)}{n B}
$$

where $B$ is defined in appendices.

\subsection{Four stage and multi-stage adaptive models}

Extending the notion in the above sub-sections further we have estimators at stages four and $n$ given by solutions to

$$
\begin{aligned}
& \frac{k_{1} X_{1} q^{k_{1}}[(1-\phi)-\eta]}{\eta-(\eta+(1-\phi)) q^{k_{1}}}+\frac{k_{2}\left(X_{1}\right) X_{2} q^{k_{2}\left(X_{1}\right)}[(1-\phi)-\eta]}{\eta-(\eta+(1-\phi)) q^{k_{2}\left(X_{1}\right)}} \\
& +\frac{k_{3}\left(X_{1}, X_{2}\right) X_{3} q^{k_{3}\left(X_{1}, X_{2}\right)}[(1-\phi)-\eta]}{\eta-(\eta+(1-\phi)) q^{k_{3}\left(X_{1}, X_{2}\right)}}+\frac{k_{4}\left(X_{1}, X_{2}, X_{3}\right) X_{4} q^{k_{4}\left(X_{1}, X_{2}, X_{3}\right)}[(1-\phi)-\eta]}{\eta-(\eta+(1-\phi)) q^{k_{4}\left(X_{1}, X_{2}, X_{3}\right)}} \\
& =\frac{k_{1} q^{k_{1}}\left(\lambda_{1} n-X_{1}\right)(\eta+(1-\phi))}{1-\left[\eta-(\eta+(1-\phi)) q^{k_{1}}\right]}+\frac{k_{2}\left(X_{1}\right) q^{k_{2}\left(X_{1}\right)}\left[\lambda_{2} n-X_{2}\right][\eta+(1-\phi)]}{1-\left[\eta-(\eta+(1-\phi)) q^{k_{2}\left(X_{1}\right)}\right]} \\
& +\frac{k_{3}\left(X_{1}, X_{2}\right) q^{k_{3}\left(X_{1}, X_{2}\right)}\left[\lambda_{3} n-X_{3}\right][\eta+(1-\phi)]}{1-\left[\eta-(\eta+(1-\phi)) q^{k_{3}\left(X_{1}, X_{2}\right)}\right]} \\
& +\frac{k_{4}\left(X_{1}, X_{2}, X_{3}\right) q^{k_{4}\left(X_{1}, X_{2}, X_{3}\right)}\left[\left(1-\lambda_{1}-\lambda_{2}-\lambda_{3}\right) n-X_{4}\right][\eta+(1-\phi)]}{1-\left[\eta-(\eta+(1-\phi)) q^{k_{4}\left(X_{1}, X_{2}, X_{3}\right)}\right]}=0
\end{aligned}
$$

and

$$
\begin{aligned}
& \frac{k_{1} X_{1} q^{k_{1}}[(1-\phi)-\eta]}{\eta-(\eta+(1-\phi)) q^{k_{1}}}+\frac{k_{2}\left(X_{1}\right) X_{2} q^{k_{2}\left(X_{1}\right)}[(1-\phi)-\eta]}{\eta-(\eta+(1-\phi)) q^{k_{2}\left(X_{1}\right)}} \ldots \\
& +\frac{\left.k_{n}\left(X_{1}, \ldots, X_{(} n-1\right)\right) X_{n} q^{k_{n}\left(X_{1}, \ldots, X_{(}(n-1)\right)}[(1-\phi)-\eta]}{\eta-(\eta+(1-\phi)) q^{k_{n}\left(X_{1}, \ldots, X_{(}(n-1)\right)}} \\
= & \frac{k_{1} q^{k_{1}}\left(\lambda_{1} n-X_{1}\right)(\eta+(1-\phi))}{1-\left[\eta-(\eta+(1-\phi)) q^{k_{1}}\right]}+\frac{k_{2}\left(X_{1}\right) q^{k_{2}\left(X_{1}\right)}\left[\lambda_{2} n-X_{2}\right][\eta+(1-\phi)]}{1-\left[\eta-(\eta+(1-\phi)) q^{k_{2}\left(X_{1}\right)}\right]} \ldots \\
& +\frac{\left.k_{n}\left(X_{1}, \ldots, X_{(} n-1\right)\right) q^{\left.k_{n}\left(X_{1}, \ldots, X_{(} n-1\right)\right)}\left[\left(1-\lambda_{1}-\lambda_{2}\right) n-X_{4}\right][\eta+(1-\phi)]}{1-\left[\eta-(\eta+(1-\phi)) q^{k_{n}\left(X_{1}, \ldots, X_{(}(n-1)\right)}\right]}=0,
\end{aligned}
$$

respectively. Using Cramer-Rao lower bound method their variances are obtained as

$$
\operatorname{Var}\left(\hat{p}_{4}\right)=\frac{\pi_{1}(p) \pi_{2}(p) \pi_{3}(p) \pi_{4}(p)\left(1-\pi_{1}(p)\right)(p)\left(1-\pi_{2}(p)\right)(p)\left(1-\pi_{3}(p)\right)\left(1-\pi_{4}(p)\right)}{C}
$$

and

$$
\operatorname{Var}\left(\hat{p}_{n}\right)=\frac{\pi_{1}(p) \pi_{2}(p) \ldots \pi_{n}(p)\left(1-\pi_{1}(p)\right)(p)\left(1-\pi_{2}(p)\right)(p) \ldots\left(1-\pi_{n}(p)\right)}{D}
$$

where $C$ and $D$ are given in the appendices respectively.

\subsection{Confidence Interval(CI) of $\hat{p}_{n}$}

Next we provide the confidence interval for our multi-stage estimator, $\hat{p}_{n}$. This confidence interval is given by

$$
\hat{p}_{n}^{+} Z_{\frac{\alpha}{2}} \sqrt{\operatorname{var}\left(\hat{p}_{n}\right)}
$$

where $Z_{\frac{\alpha}{2}} \sim \operatorname{Normal}(0,1)$. and $\hat{p}_{n}$ and $\operatorname{var}\left(\hat{p}_{n}\right)$ are provided by the solution to 15 and Equation 17 respectively. It follows from Equation 18 that

$$
p \in\left[\hat{p}_{n}-Z_{\frac{\alpha}{2}} \sqrt{\operatorname{var}\left(\hat{p}_{n}\right)}, \hat{p}_{n}+Z_{\frac{\alpha}{2}} \sqrt{\operatorname{var}\left(\hat{p}_{n}\right)}\right]
$$


and by the law of Central Limit Theorem (CLT) we have

$$
\sqrt{n}\left(\hat{p}_{n}-p\right) \stackrel{l}{\rightarrow} \operatorname{Normal}\left(0, \sqrt{\operatorname{var}\left(\hat{p}_{n}\right)}\right)
$$

or

$$
\sqrt{n} \frac{\left(\hat{p}_{n}-p\right)}{\left.\sqrt{\operatorname{var}\left(\hat{p}_{n}\right)}\right)} \stackrel{l}{\rightarrow} \operatorname{Normal}(0,1)
$$

\section{Discussion of Results, Conclusion and Recommendations}

In this section we discuss the results as provided by Tables 4.1, 4.2 and 4.3 and Figures 4.1, 4.2 and 4.3. The highlights of the results will enable us make a detailed conclusion to this study.

\subsection{Discussion}

Here we highlight our findings in this study. We estimated prevalence, $p$ of a trait using the Multi-stage adaptive pool testing scheme. We accomplished this by employing the Maximum Likelihood Estimate (MLE) procedure. For us to recommend the suitability of the Multi-stage adaptive estimator, it would be in order to first compare with the non-adaptive estimator in the presence of test errors, as advanced by [7]; and then do an inter-stage comparison. Our measure of comparison herein is the computation of Asymptotic Relative Efficiency (ARE) values for different values of $\eta$ and $\phi$ at various stages. For simplicity of comparison and understanding, ARE values were computed for stages two to four. Upon careful analysis of the estimators at these stages, we had a good basis to make a generalization about the multi-stage estimator in the presence of test errors.

4.1.1 Comparing the adaptive estimators with the non-adaptive estimator in the presence of test errors

The ARE values for stages two, three and four were obtained by dividing Equations (5) by (9), (13) and (16) respectively. Upon simplification we obtained

$$
A R E_{\hat{p}_{2}}=\frac{H}{\pi_{1}(p) \pi_{2}(p)\left(1-\pi_{1}(p)\right)\left(1-\pi_{2}(p)\right)(1-p)^{2 k}},
$$

where $H$ is defined in the appendices. Similarly, the Asymptotic Relative efficiencies of $\hat{p}_{3}$ and $\hat{p}_{4}$ are given as

$$
A R E_{\hat{p}_{3}}=\frac{J}{\pi_{1}(p) \pi_{2}(p) \pi_{3}\left(1-\pi_{1}(p)\right)\left(1-\pi_{2}(p)\right)\left(1-\pi_{3}(p)\right)(1-p)^{2 k}},
$$

and

$$
A R E_{\hat{p}_{4}}=\frac{L}{\pi_{1}(p) \pi_{2}(p)\left(1-\pi_{1}(p)\right)\left(1-\pi_{2}(p)\right)(1-p)^{2 k}},
$$

respectively, where $J$ and $L$ are defined in the appendices. respectively. Using these Equations and R-Gui software Tables 4.1, 4.2 and 4.3 were generated. 
Multi-Stage Adaptive Pool Testing Model with Test Errors; Improved Efficiency

\begin{tabular}{|c|c|c|c|c|c|}
\hline$p$ & $\eta=\phi=0.99$ & $\eta=\phi=0.98$ & $\eta=\phi=0.97$ & $\eta=\phi=0.96$ & $\eta=\phi=0.90$ \\
\hline 0.1 & 11.7739 & 11.8024 & 11.8304 & 11.8577 & 12.0095 \\
\hline 0.2 & 6.1184 & 6.4223 & 6.7152 & 6.9974 & 8.4904 \\
\hline 0.3 & 3.6109 & 4.2835 & 4.8918 & 5.4448 & 7.9386 \\
\hline 0.4 & 3.0823 & 4.3658 & 5.3725 & 6.1880 & 9.0662 \\
\hline 0.5 & 4.4239 & 6.3134 & 7.4865 & 8.3030 & 10.5715 \\
\hline 0.6 & 7.3875 & 9.0611 & 9.9042 & 10.4289 & 11.6667 \\
\hline 0.7 & 10.2335 & 11.1440 & 11.5420 & 11.7672 & 12.2284 \\
\hline 0.8 & 11.9115 & 12.1858 & 12.2856 & 12.3372 & 12.4329 \\
\hline 0.9 & 12.4422 & 12.4616 & 12.4681 & 12.4713 & 12.4772 \\
\hline
\end{tabular}

Table 4.1: ARE values of $\hat{p}_{2}$ relative to $\hat{p}$ for specified $p, \eta$ and $\phi$

\begin{tabular}{|c|c|c|c|c|c|}
\hline$p$ & $\eta=\phi=0.99$ & $\eta=\phi=0.98$ & $\eta=\phi=0.97$ & $\eta=\phi=0.96$ & $\eta=\phi=0.90$ \\
\hline 0.1 & 22.7824 & 22.8485 & 22.9133 & 22.9766 & 23.3267 \\
\hline 0.2 & 15.3291 & 15.8547 & 16.3494 & 16.8157 & 19.1305 \\
\hline 0.3 & 11.6186 & 13.0352 & 14.2083 & 15.2006 & 19.0300 \\
\hline 0.4 & 11.6141 & 14.2989 & 16.0410 & 17.2898 & 20.9478 \\
\hline 0.5 & 15.4718 & 18.2260 & 19.6402 & 20.5355 & 22.7513 \\
\hline 0.6 & 200354 & 21.6224 & 22.3539 & 22.7912 & 23.7781 \\
\hline 0.7 & 22.7669 & 23.4427 & 23.7314 & 23.8933 & 24.2221 \\
\hline 0.8 & 24.0129 & 24.0000 & 24.2679 & 24.3030 & 24.3680 \\
\hline 0.9 & 24.3747 & 24.3878 & 24.3920 & 24.3942 & 24.3981 \\
\hline
\end{tabular}

Table 4.2: ARE values of $\hat{p}_{3}$ relative to $\hat{p}$ for specified $p, \eta$ and $\phi$

\begin{tabular}{|c|c|c|c|c|c|}
\hline$p$ & $\eta=\phi=0.99$ & $\eta=\phi=0.98$ & $\eta=\phi=0.97$ & $\eta=\phi=0.96$ & $\eta=\phi=0.90$ \\
\hline 0.1 & 43.4486 & 43.5040 & 43.5580 & 43.6109 & 43.9034 \\
\hline 0.2 & 37.5497 & 37.9749 & 38.3745 & 38.7509 & 40.6123 \\
\hline 0.3 & 34.5777 & 35.7352 & 36.6908 & 37.4967 & 40.5809 \\
\hline 0.4 & 34.6078 & 36.8073 & 38.2250 & 39.2350 & 42.1479 \\
\hline 0.5 & 37.8059 & 40.0398 & 41.1693 & 41.8752 & 43.5804 \\
\hline 0.6 & 41.5386 & 42.7721 & 43.3239 & 43.6477 & 44.3618 \\
\hline 0.7 & 43.6724 & 44.1484 & 44.3474 & 44.4580 & 44.6805 \\
\hline 0.8 & 44.5490 & 44.6706 & 44.7145 & 44.7373 & 44.7793 \\
\hline 0.9 & 44.7841 & 44.7922 & 44.7950 & 44.7967 & 44.7988 \\
\hline
\end{tabular}

Table 4.3: ARE values of $\hat{p}_{4}$ relative to $\hat{p}$ for specified $p, \eta$ and $\phi$

Tables 4.1, 4.2 and 4.3 provide generated ARE values for given $p, \eta$ and $\phi$. It is evident from the tables that ARE values are high across all stages. That is all the ARE values are greater than one, meaning all the adaptive estimators analysed in this study, $\hat{p}_{2}, \hat{p}_{3}$ and $\hat{p}_{4}$ are more efficient than the non-adaptive estimator in the presence of test errors. A closer look at ARE values reveals that ARE is high when $p$ is small and decreases as $p$ increases, attaining the minimum at $p=0.3$ across the board, except for $\eta=\phi=0.99$ where the minimum is attained at $p=0.4$. The ARE again improves as $p$ moves away from 0.3 for $\eta, \phi<0.99$. A similar scenario is observed for the case of $\eta=\phi=0.99$ where ARE improves as $p$ moves away from 0.4. It is also clear from the tables that ARE values increase with increase in the number of stages; the adaptive estimator at stage two having the lowest ARE values while the estimator at stage four has the highest ARE values. This is an important pointer to the fact that the adaptive testing scheme gets better as the number of stages increases. To depict these observations graphically, see Figures 4.1, 4.2 and 4.3 


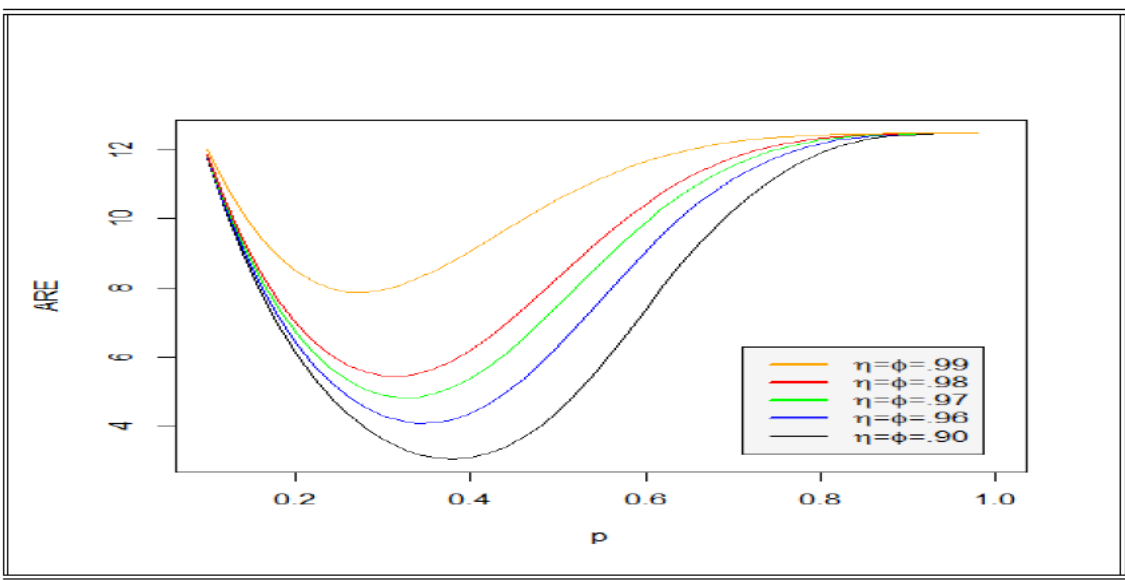

Figure 4.1: ARE of $\hat{p}_{2}$ vs probability, $p$

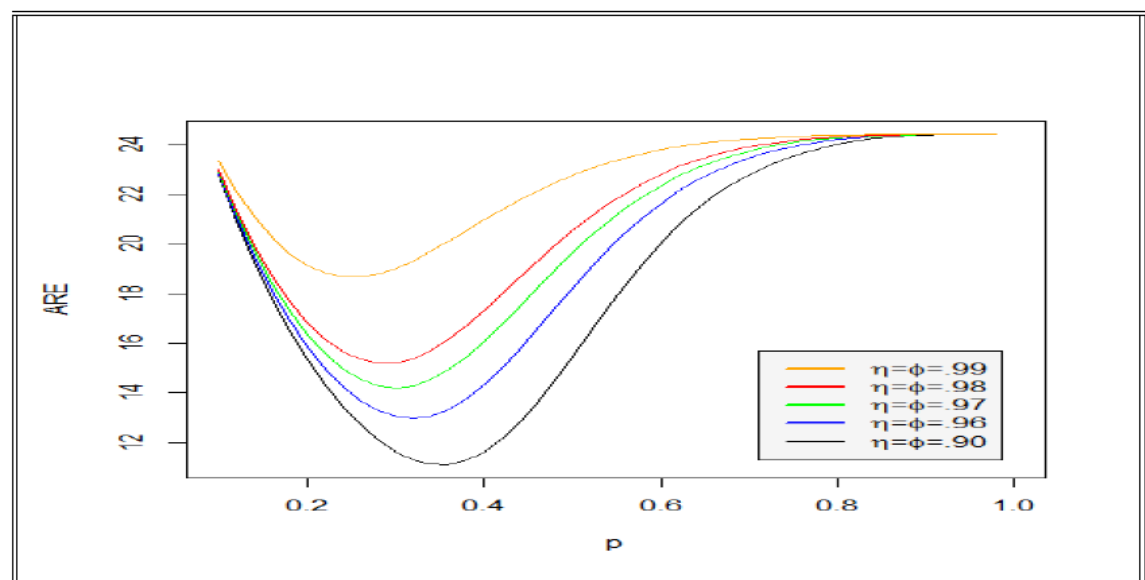

Figure 4.2: ARE of $\hat{p}_{3}$ vs probability, $p$

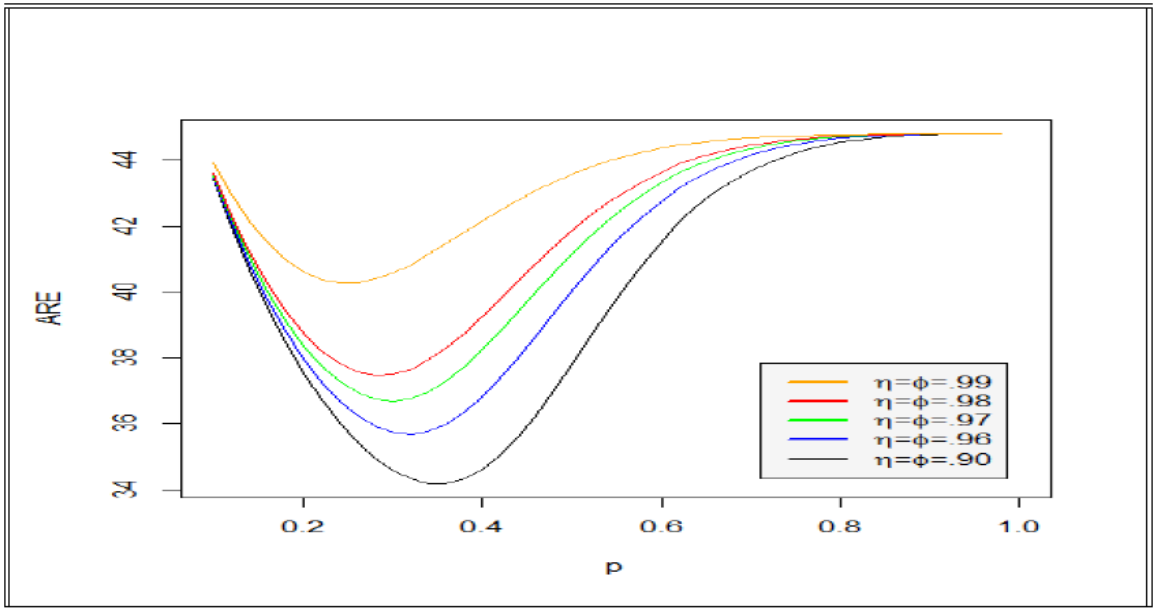

Figure 4.3: ARE of $\hat{p}_{4}$ vs probability, $p$

Figures 4.1, 4.2 and 4.3 represent ARE values plotted against prevalence $p$ values at stages two, three and four respectively. Clearly, as noted in Tables 4.1, 4.2 and 4.3, the ARE drops as $p$ increases up to the value $p=0.3$, then it improves as $p$ moves away from 0.3 . From

Figures 4.1, 4.2 and 4.3, it is evident that the adaptive estimators outperform the non-adaptive estimator in the presence of test errors as the sensitivity and specificity of the test kit decreases. Hence in cases where the test kits have low sensitivity and specificity, the multi-stage adaptive testing scheme is preferred for more efficient results. 


\subsubsection{Comparing the adaptive estimators at various stages}

Next we compared Asymptotic Relative Efficiencies among the constructed estimators in this study. Here we compared $\hat{p}_{2}$ with $\hat{p}_{1}, \hat{p}_{3}$ with $\hat{p}_{2}$ and $\hat{p}_{4}$ with $\hat{p}_{3}$. This analysis would enable us to make important generalizations about the efficiency of the multi-stage adaptive estimator, $\hat{p}_{n}$. ARE values for adaptive estimators at stages two, three and four relative to adaptive estimators at stages one, two and three respectively were obtained by dividing Equation (5), where $\hat{p}=\hat{p}_{1}$ and $k=k_{1}$ by Equation (9), Equation (9) by Equation (13) and Equation (13) by Equation (16) respectively.

$$
\frac{\operatorname{Var}\left(\hat{p}_{1}\right)}{\operatorname{Var}\left(\hat{p}_{2}\right)}, \frac{\operatorname{Var}\left(\hat{p}_{2}\right)}{\operatorname{Var}\left(\hat{p}_{3}\right)} \text { and } \frac{\operatorname{Var}\left(\hat{p}_{3}\right)}{\operatorname{Var}\left(\hat{p}_{4}\right)},
$$

Upon simplification we obtain

$$
\begin{gathered}
A R E_{\hat{p}_{2}}=\lambda k_{1}^{2}+\frac{\pi_{1}\left(1-\pi_{1}\right)(1-\lambda) k_{2}^{2}(1-p)^{2 k_{2}}}{(1-p)^{2 k_{1}} \pi_{2}\left(1-\pi_{2}\right)} \\
A R E_{\hat{p}_{3}}=\frac{M}{\pi_{2}\left(1-\pi_{2}\right) \lambda k_{1}^{2}(1-p)^{2 k_{1}-2}+\pi_{1}\left(1-\pi_{1}\right)(1-\lambda) k_{2}^{2}(1-p)^{2 k_{2}-2}}
\end{gathered}
$$

and

$$
A R E_{\hat{p}_{4}}=\frac{N}{R}
$$

where $M, N$ and $R$ are defined in the appendices. Using these Equations and R-Gui software Tables $4.4,4.5$ and 4.6 were generated.

\begin{tabular}{|c|c|c|c|c|c|}
\hline$p$ & $\eta=\phi=0.99$ & $\eta=\phi=0.98$ & $\eta=\phi=0.97$ & $\eta=\phi=0.96$ & $\eta=\phi=0.90$ \\
\hline 0.1 & 9.7163 & 9.7158 & 9.7153 & 9.7149 & 9.7126 \\
\hline 0.2 & 9.7369 & 9.7383 & 9.7396 & 9.7409 & 9.7480 \\
\hline 0.3 & 9.7712 & 9.7775 & 9.7836 & 9.7893 & 9.8188 \\
\hline 0.4 & 9.8384 & 9.8627 & 9.8846 & 9.9043 & 9.9913 \\
\hline 0.5 & 10.0142 & 10.1098 & 10.1865 & 10.2492 & 10.4700 \\
\hline 0.6 & 10.7070 & 11.0878 & 11.3347 & 11.5076 & 11.9708 \\
\hline 0.7 & 14.7585 & 16.0357 & 16.6444 & 17.0000 & 17.7520 \\
\hline 0.8 & 47.4125 & 50.0919 & 51.0753 & 51.5856 & 52.5348 \\
\hline
\end{tabular}

Table 4.4: ARE values of $\hat{p}_{2}$ relative to $\hat{p}_{1}$ for specified $p, \eta$ and $\phi$

\begin{tabular}{|c|c|c|c|c|c|}
\hline$p$ & $\eta=\phi=0.99$ & $\eta=\phi=0.98$ & $\eta=\phi=0.97$ & $\eta=\phi=0.96$ & $\eta=\phi=0.90$ \\
\hline 0.1 & 3.6211 & 3.6160 & 3.6111 & 3.6063 & 3.5801 \\
\hline 0.2 & 6.7871 & 6.6413 & 6.5045 & 6.3761 & 5.7484 \\
\hline 0.3 & 17.6647 & 16.0685 & 14.7652 & 13.6813 & 9.7387 \\
\hline 0.4 & 48.5664 & 37.0247 & 30.0569 & 25.3924 & 13.6778 \\
\hline 0.5 & 102.7462 & 61.4910 & 44.1744 & 34.6400 & 15.7793 \\
\hline 0.6 & 140.1473 & 73.3601 & 50.0271 & 38.1510 & 16.4663 \\
\hline 0.7 & 150.1342 & 76.1234 & 51.3380 & 38.9296 & 16.6292 \\
\hline 0.8 & 151.4241 & 76.4962 & 51.5258 & 39.0479 & 16.6611 \\
\hline
\end{tabular}

Table 4.5: ARE values of $\hat{p}_{3}$ relative to $\hat{p}_{2}$ for specified $p, \eta$ and $\phi$ 


\begin{tabular}{|c|c|c|c|c|c|}
\hline$p$ & $\eta=\phi=0.99$ & $\eta=\phi=0.98$ & $\eta=\phi=0.97$ & $\eta=\phi=0.96$ & $\eta=\phi=0.90$ \\
\hline 0.1 & 3.9481 & 3.9349 & 3.9221 & 3.9096 & 3.8420 \\
\hline 0.2 & 9.8857 & 9.4430 & 9.0475 & 8.6920 & 7.1475 \\
\hline 0.3 & 31.9137 & 26.4622 & 22.6951 & 19.9353 & 11.9794 \\
\hline 0.4 & 85.1181 & 54.4296 & 40.3510 & 32.2515 & 15.3742 \\
\hline 0.5 & 135.2677 & 72.6353 & 50.1207 & 38.4952 & 16.8860 \\
\hline 0.6 & 154.7372 & 79.1945 & 53.6152 & 40.7423 & 17.4759 \\
\hline 0.7 & 160.3179 & 81.1190 & 54.6702 & 41.44267 & 17.6922 \\
\hline 0.8 & 161.4478 & 81.5535 & 54.9309 & 41.6278 & 17.7616 \\
\hline
\end{tabular}

Table 4.6: ARE values of $\hat{p}_{4}$ relative to $\hat{p}_{3}$ for specified $p, \eta$ and $\phi$

As clearly shown from tables 4.4, 4.5 and 4.6, ARE values are high across all stages. That is, the adaptve estimator at stage two is is more efficient than that at stage one, the adaptive estimator at stage three is more efficient than that at stage two and the estimator at stage four is more efficient than that at stage three. This means that $\hat{p}_{4}$ is a better estimator than $\hat{p}_{3}, \hat{p}_{3}$ is a better estimator than $\hat{p}_{2}$ and $\hat{p}_{2}$ is a better estimator than $\hat{p}_{1}$. A closer look at table 4.4 shows that ARE values for $\hat{p}_{2}$ relative to $\hat{p}_{1}$ rise by very small margins at values of $p<0.8$ and steadily shoot when $p=0.8$, while for higher estimators ARE values rise steadily at $p<0.6$ and almost level off at $p=0.6,0.7,0.8$. It is also evident from tables 4.5 and 4.6 that ARE values increase with increase in sensitivity and specificity of the test kits. As pointed out earlier, this analysis shows that the adaptive testing scheme improves as the number of stages increases.

These observations can be graphically depicted by Figures $4.4,4.5$ and 4.6 .

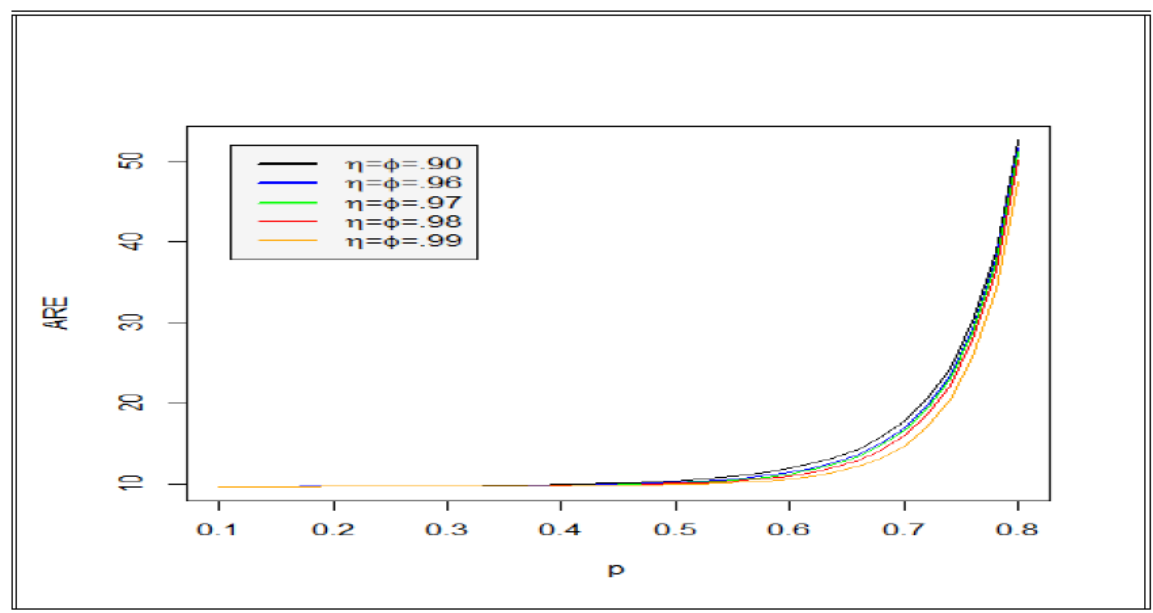

Figure 4.4: ARE of $\hat{p}_{2}$ relative to $\hat{p}_{1}$ vs probability, $p$

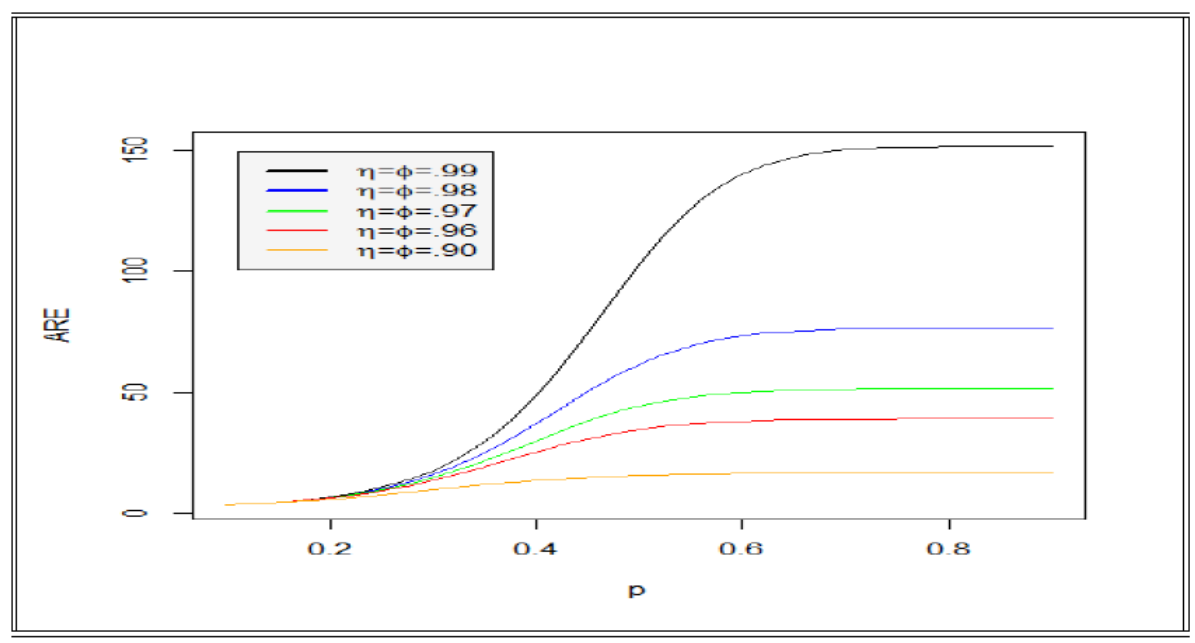

Figure 4.5: ARE of $\hat{p}_{3}$ relative to $\hat{p}_{2}$ vs probability, $p$ 


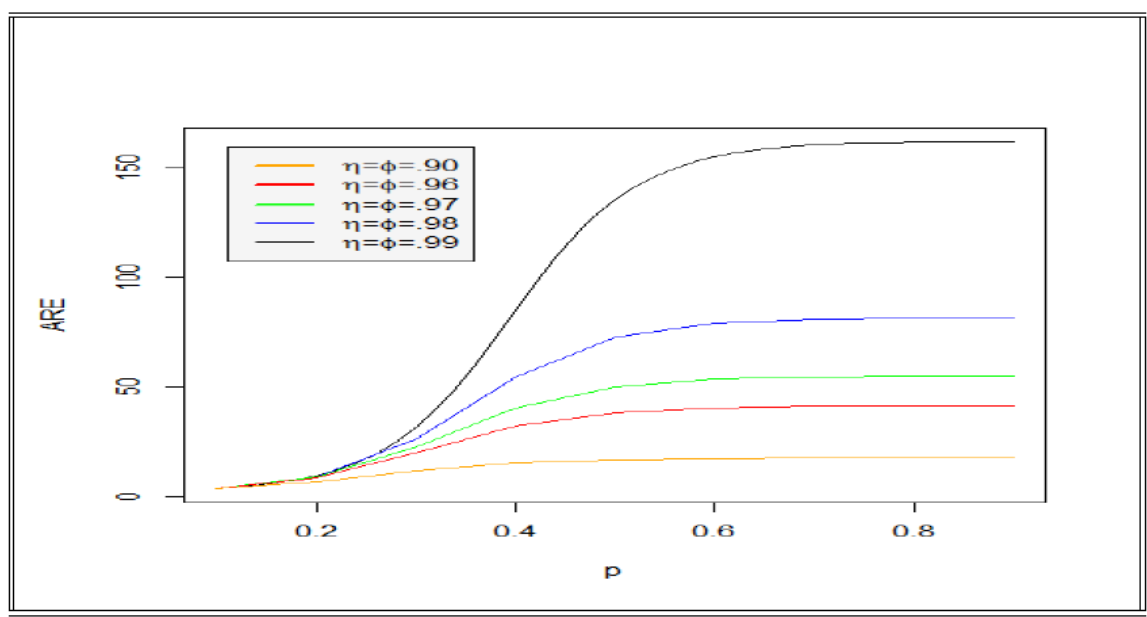

Figure 4.6: ARE of $\hat{p}_{4}$ relative to $\hat{p}_{3}$ vs probability, $p$

\subsubsection{Conclusion and Recommendations}

From the above discussions, it is clear that the multi-stage adaptive estimator outperforms the non-adaptive estimator in the presence of test errors. It is also clear that the adaptive testing scheme improves as the number of stages increases. A closer look at the results reveals that the multi-stage adaptive estimator is particularly better in cases where test kits have low sensitivity and specificity. Given that experiments are never $100 \%$ perfect, the multi-stage adaptive testing scheme is therefore more ideal in estimating prevalence of a trait.

\section{References}

[1] Bhattacharyya, G.K., Karandinos, M.G., and DeFoliart, G. R. (1979). Point estimates and confidence intervals for infection rates using pooled organisms in epidemiologic studies. American journal of epidemiology 109,124-131.

[2] Brookmeyer, R. (1999). Analysis of multistage pooling studies of biological specimens for estimating disease incidence and prevalence. Biometrics 55,608-612.

[3] Chiang, C. L. and Reeves, W. C. (1962). Statistical estimation of virus infection rates in mosquito vector populations. American journal on hygiene 75, 377-391

[4] Dorfman R. (1943). The detection of defective members of large populations. Annals of mathematical statistics 14, 436-440.

[5] Gastwirth, J.L., and Hammick,P.A. (1989). Estimation of prevalence of a rare disease, preserving the anonymity of the subjects by group testing: Application to estimate the prevalence of AIDS antibodies in blood donors. Journal of Statistical Planning and Inference 22, $15-27$.

[6] Mood, D. A., Graybill, G. A., Boes, D. C. (1974). Introduction to the theory of statistics. 27,72 .

[7] Nyongesa, L. K. (2011). Dual estimation of prevalence and disease incidence in pool testing strategy. Communication in statistics theory and methods, 40, 1-12

[8] Okoth, A. W. (2012). Two Stage Adaptive Pool Testing for estimating prevalence of a trait in the presence of test errors. Lambert Academic publishers, 18 and 27

[9] Oliver-Hughes J.M. and Swallow W.H. (1994), A two-stage adaptive group testing procedure for estimating small proportions. American statistical association 89, 982-993.

[10] Richards, M. S. (1991). Interpretation of the results of bacteriological testing of egg laying flocks for salmonella enteritidis. Proceedings of the 6 th international symposium on veterinary epidemiology and economics, 124-126, Ottawa, Canada.

[11] Sobel M., Ellashoff R.M. (1975). Group testing with a new goal; estimation. Biometrika 62, 181-193.

[12] Swallow W.H. (1987).Relative MSE and cost considerations in choosing group size for group testing to estimate infection rates and probability of disease infection. Phytopathology $\mathbf{7 7}$, 1376-1381. 


\section{Appendices}

$$
\begin{aligned}
A= & (\eta+\phi-1)^{2} n \\
& {\left[\pi_{2}(p)\left(1-\pi_{2}(p)\right) \lambda k_{1}^{2}(1-p)^{2 k_{1}-2}+\pi_{1}(p)\left(1-\pi_{1}(p)\right)(1-\lambda) k_{2}^{2}\left(X_{1}\right)(1-p)^{2 k_{2}\left(X_{1}\right)-2}\right] } \\
& \\
& \quad=\quad(\eta+\phi-1)^{2} \\
& \quad * \quad\left[\pi_{2}(p)\left(1-\pi_{2}(p)\right) \pi_{3}(p)\left(1-\pi_{3}(p)\right) \lambda_{1} n k_{1}(1-p)^{k_{1}-2}\right. \\
& \quad+\pi_{1}(p)\left(1-\pi_{1}(p)\right) \pi_{3}(p)\left(1-\pi_{3}(p)\right) \lambda_{2} n k_{2}\left(X_{1}\right)(1-p)^{k_{2}\left(X_{1}\right)-2} \\
& \quad+\pi_{1}(p)\left(1-\pi_{1}(p)\right) \pi_{2}(p)\left(1-\pi_{2}(p)\right) \lambda_{2} n k_{3}\left(X_{2}\right)(1-p)^{k_{3}\left(X_{2}\right)-2} \\
& \\
C & (\eta+\phi-1)^{2} n \\
& {\left[\pi_{2}(p)\left(1-\pi_{2}(p)\right) \pi_{3}(p)\left(1-\pi_{3}(p)\right) \pi_{4}(p)\left(1-\pi_{4}(p)\right) \lambda_{1} n k_{1}(1-p)^{k_{1}-2}\right.} \\
+ & \pi_{1}(p)\left(1-\pi_{1}(p)\right) \pi_{3}(p)\left(1-\pi_{3}(p)\right) \pi_{4}(p)\left(1-\pi_{4}(p)\right) \lambda_{2} n k_{2}\left(X_{1}\right)(1-p)^{k_{2}\left(X_{1}\right)-2} \\
+ & \pi_{1}(p)\left(1-\pi_{1}(p)\right) \pi_{2}(p)\left(1-\pi_{2}(p)\right) \pi_{4}(p)\left(1-\pi_{4}(p)\right) \lambda_{3} n k_{3}\left(X_{2}\right)(1-p)^{k_{3}\left(X_{2}\right)-2} \\
+ & \pi_{1}(p)\left(1-\pi_{1}(p)\right) \pi_{2}(p)\left(1-\pi_{2}(p)\right) \pi_{3}(p)\left(1-\pi_{3}(p)\right)\left(1-\lambda_{1}-\lambda_{2}-\lambda_{3}\right) n k_{4}\left(X_{3}\right)(1-p)^{k_{4}\left(X_{3}\right)-2}
\end{aligned}
$$

$$
\begin{aligned}
& D=(\eta+\phi-1)^{2} n \\
& \text { * }\left[\pi_{2}(p)\left(1-\pi_{2}(p)\right) \ldots \pi_{n}(p)\left(1-\pi_{n}(p)\right) \lambda_{1} n k_{1}(1-p)^{k_{1}-2}\right. \\
& +\pi_{1}(p)\left(1-\pi_{1}(p)\right) \ldots \pi_{n}(p)\left(1-\pi_{n}(p)\right) \lambda_{2} n k_{2}\left(X_{1}\right)(1-p)^{k_{2}\left(X_{1}\right)-2}+\ldots . \\
& \left.+\pi_{1}(p)\left(1-\pi_{1}(p)\right) \pi_{2}(p)\left(1-\pi_{2}(p)\right) \ldots . . \pi_{(} n-1\right)(p)\left(1-\pi_{(n-1)(p))}\right. \\
& \left.\left.\left.\left(1-\lambda_{1}-\lambda_{2}-\ldots-\lambda_{(} n-1\right)\right) n k_{n}\left(X_{(} n-1\right)\right)(1-p)^{k_{n}\left(X_{3}\right)-2}\right] \\
& H=\pi(p)(1-\pi(p)) \\
& \text { * }\left[\pi_{2}(p)\left(1-\pi_{2}(p)\right) \lambda k_{1}^{2}(1-p)^{2 k_{1}}+\pi_{1}(p)\left(1-\pi_{1}(p)\right)(1-\lambda) k_{2}^{2}\left(X_{1}\right)(1-p)^{2 k_{2}\left(X_{1}\right)}\right] \\
& J=\pi(p)(1-\pi(p)) \\
& \text { * }\left[\pi_{2}(p) \pi_{3}(p)\left(1-\pi_{2}(p)\right)\left(1-\pi_{3}(p)\right) \lambda_{1} k_{1}^{2}(1-p)^{2 k_{1}}\right. \\
& +\pi_{1}(p) \pi_{3}(p)\left(1-\pi_{1}(p)\right)\left(1-\pi_{3}(p)\right) \lambda_{2} k_{2}^{2}\left(X_{1}\right)(1-p)^{2 k_{2}\left(X_{1}\right)} \\
& \left.+\pi_{1}(p) \pi_{2}(p)\left(1-\pi_{1}(p)\right)\left(1-\pi_{2}(p)\right)\left(1-\lambda_{1}-\lambda_{2}\right) k_{3}^{2}\left(X_{2}\right)(1-p)^{2 k_{3}\left(X_{2}\right)}\right] \\
& L=\pi(p)(1-\pi(p)) \\
& \text { * }\left[\pi_{2}(p) \pi_{3} \pi_{4}(p)\left(1-\pi_{2}(p)\right)\left(1-\pi_{3}(p)\right)\left(1-\pi_{4}(p)\right) \lambda_{1} k_{1}^{2}(1-p)^{2 k_{1}}\right. \\
& +\pi_{1}(p) \pi_{3}(p) \pi_{4}\left(1-\pi_{1}(p)\right)\left(1-\pi_{3}(p)\right)\left(1-\pi_{4}(p)\right) \lambda_{2} k_{2}^{2}\left(X_{1}\right)(1-p)^{2 k_{2}\left(X_{1}\right)} \\
& +\pi_{1}(p) \pi_{2}(p) \pi_{4}(p)\left(1-\pi_{1}(p)\right)\left(1-\pi_{2}(p)\right)\left(1-\pi_{4}(p)\right) \lambda_{3} k_{3}^{2}\left(X_{2}\right)(1-p)^{2 k_{3}\left(X_{2}\right)} \\
& \left.+\pi_{1}(p) \pi_{2}(p) \pi_{3}(p)\left(1-\pi_{1}(p)\right)\left(1-\pi_{2}(p)\right)\left(1-\pi_{3}(p)\right)\left(1-\lambda_{1} \lambda_{2} \lambda_{3}\right) k_{4}^{2}\left(X_{3}\right)(1-p)^{2 k_{4}\left(X_{3}\right)}\right]
\end{aligned}
$$




$$
\begin{aligned}
& M=\pi_{2} \pi_{3}\left(1-\pi_{2}\right)\left(1-\pi_{3}\right) \lambda_{1} k_{1}^{2}(1-p)^{2 k_{1}-2} \\
& +\pi_{1} \pi_{3}\left(1-\pi_{1}\right)\left(1-\pi_{3}\right) \lambda_{2} k_{2}^{2}(1-p)^{2 k_{2}-2} \\
& +\pi_{1} \pi_{2}\left(1-\pi_{1}\right)\left(1-\pi_{2}\right)\left(1-\lambda_{1}-\lambda_{2}\right) k_{3}^{2}(1-p)^{2 k_{3}-2} \\
& N=\pi_{2} \pi_{3} \pi_{4}\left(1-\pi_{2}\right)\left(1-\pi_{3}\right)\left(1-\pi_{4}\right) \lambda_{1} k_{1}^{2}(1-p)^{2 k_{1}-2} \\
& +\pi_{1} \pi_{3} \pi_{4}\left(1-\pi_{1}\right)\left(1-\pi_{3}\right)\left(1-\pi_{4}\right) \lambda_{2} k_{2}{ }^{2}(1-p)^{2 k_{2}-2} \\
& \left.+\pi_{1} \pi_{2} \pi_{4}\left(1-\pi_{1}\right)\left(1-\pi_{2}\right)\left(1-\pi_{4}\right) \lambda_{3}\right) k_{3}{ }^{2}(1-p)^{2 k_{3}-2} \\
& +\pi_{1} \pi_{2} \pi_{3}\left(1-\pi_{1}\right)\left(1-\pi_{2}\right)\left(1-\pi_{3}\right)\left(1-\lambda_{1}-\lambda_{2}-\lambda_{3}\right) k_{4}^{2}(1-p)^{2 k_{4}-2} \\
& \begin{aligned}
R & =\pi_{2} \pi_{3}\left(1-\pi_{2}\right)\left(1-\pi_{3}\right) \lambda_{1} k_{1}^{2}(1-p)^{2 k_{1}-2} \\
& +\pi_{1} \pi_{3}\left(1-\pi_{1}\right)\left(1-\pi_{3}\right) \lambda_{2}{k_{2}}^{2}(1-p)^{2 k_{2}-2} \\
& +\pi_{1} \pi_{2}\left(1-\pi_{1}\right)\left(1-\pi_{2}\right)\left(1-\lambda_{1}-\lambda_{2}\right) k_{3}{ }^{2}(1-p)^{2 k_{3}-2}
\end{aligned}
\end{aligned}
$$

